University of Nebraska - Lincoln

DigitalCommons@University of Nebraska - Lincoln

Timothy J. Gay Publications

Research Papers in Physics and Astronomy

6-1-1993

\title{
High-Voltage Variable Resistor for Ion Energy Spectroscopy
}

Timothy J. Gay

University of Nebraska - Lincoln, tgay1@unl.edu

V. D. Irby

University of Missouri-Rolla, Rolla, Missouri

S. P. Yallaly

University of Missouri-Rolla, Rolla, Missouri

Follow this and additional works at: https://digitalcommons.unl.edu/physicsgay

Part of the Physics Commons

Gay, Timothy J.; Irby, V. D.; and Yallaly, S. P., "High-Voltage Variable Resistor for Ion Energy Spectroscopy" (1993). Timothy J. Gay Publications. 40.

https://digitalcommons.unl.edu/physicsgay/40

This Article is brought to you for free and open access by the Research Papers in Physics and Astronomy at DigitalCommons@University of Nebraska - Lincoln. It has been accepted for inclusion in Timothy J. Gay Publications by an authorized administrator of DigitalCommons@University of Nebraska - Lincoln. 


\title{
High-voltage variable resistor for ion energy spectroscopy
}

\author{
T. J. Gay, V. D. (rby, ${ }^{\text {a) }}$ and S. P. Yallaly ${ }^{\text {b) }}$ \\ Physics Department and Laboratory for Atomic and Molecular Research. University of Missouri-Rolla, \\ Rolla, Missouri 65401
}

(Received 3 August 1992; accepted for publication 25 January 1993)

\begin{abstract}
A high-voltage variable resistor was designed, built, and implemented to modify an ion-energy spectrometer for the study of ion-atom collisions in which the projectiles change charge. The resistor is remotely switchable from 0 to $2050 \mathrm{M} \Omega$ and has a voltage rating of $200 \mathrm{kV}$. The design criteria and the electrical and mechanical details of the apparatus are discussed. The design and construction of an ancillary device, comprising two precision resistive-divider voltmeters, are also discussed.
\end{abstract}

\section{INTRODUCTION}

Projectile-ion (or "translational") energy spectroscopy is a useful method for the study of ion-atom collisions. ${ }^{1-7}$ If state-specific information about the collision products is required, its use is unavoidable when characteristic photons are not emitted by the collision partners or if such emission is not easily induced by techniques such as electric-field quenching. ${ }^{8}$ In the case of the collision

$$
\mathrm{H}^{+}+\mathrm{He}\left(1^{1} S\right) \rightarrow \mathrm{H}^{+}+\mathrm{He}^{*},
$$

for example, production cross sections for the $\operatorname{He}\left(2{ }^{1} S\right)$ state can be measured only by ion-energy-loss spectroscopy. ${ }^{9}$ Even if photons are emitted by the final state of interest, allowing cross sections to be determined using fluorescence detection, ${ }^{10}$ the energy-spectroscopic method has several advantages. ${ }^{1}$ First, the need for cascading corrections due to population of higher-lying states is eliminated because the projectile is energy analyzed and detected directly. Secondly, the same detector is used for the incident and scattered beams. Thus its efficiency $\varepsilon$ need not be known for absolute cross-section measurements, as long as the projectile does not change charge. In a chargechanging collision, the technique still yields relative cross sections for discrete states with a given final charge and at high energies, where $\varepsilon$ tends to be insensitive to charge state, ${ }^{11}$ absolute cross sections can be extracted.

An apparatus for the study of intermediate (20-200 $\mathrm{keV}$ ) energy ion-atom collisions by projectile-ion-energy. spectroscopy, developed between 1963 and 1978 in our lab, is shown in Fig. 1. 12-14 An electrical schematic of this apparatus is shown in Fig. 2. Positive ion beams with a nominal energy spread of $1 \mathrm{eV}$ are produced at high potential in the accelerator terminal. The ions are accelerated to the requisite collision energy, and are steered and focused into the target chamber, which is at ground potential. Ions emerging from the target chamber are mass analyzed and decelerated prior to electrostatic energy analysis and detection in the decelerator high-voltage terminal.

\footnotetext{
${ }^{a}$ Present address: Department of Physics and Astronomy, University of Kentucky, Lexington, KY 40506.

b) Present address: McDonnell Aircraft Company, P.O. Box 516, St. Louis, MO 63166-0516.
}

Ion-energy spectra are obtained by monitoring the ion detector signal as a function of the output voltage of a " $\Delta V$ " power supply (housed in the decelerator terminal) which, in conjunction with the main high-voltage ("HV") and " $V_{0}$ " power supplies, determines the potential of the ion source relative to ground. The electrostatic energy analyzer in the decelerator terminal will pass only ions of a given energy $E_{0}$. Ion energies immediately following a collision with the target are $q\left(V_{0}+\mathrm{HV}+\Delta V\right)-\Delta E$, where $V_{0}$ is the extraction voltage of the ion source, $H V$ is the voltage output of the main high-voltage power supply, $\Delta V$ is the output of the $\Delta V$ supply, $\Delta E$ is the energy lost in the collision, and $q$ is the ion charge. In order for an ion to be detected, its energy at ground (target) potential must equal $q(\mathrm{HV})+E_{0}$. Thus by setting $E_{0}=q V_{0}$, and $\Delta V$ $=\Delta E / q$, only ions which have lost an energy $\Delta E$ are detected, because all ions passed by the electrostatic analyzer strike the detector with energy $E_{0}$. An example of an energy-loss spectrum for reaction (1) is shown in Fig. 3.

One of the experimental goals in bullding the apparatus just described was to study collisions with targets of atomic hydrogen. Concerns about the high pumping speed and electrical current requirements of an atomic hydrogen furnace target and the large size and current requirements of an analyzing magnet for $200 \mathrm{keV}$ projectiles led to an early design decision to place the ion source and analyzer/ detector (as opposed to the target chamber) at high voltage. In this configuration the use of a common main highvoltage power supply for both the accelerator and decelerator terminals is required. In order to achieve the desired instrumental energy resolution of $\approx 1 \mathrm{eV}$, the stability of two independent accelerator and cecelerator highvoltage supplies (including both regulation and ripple) would have to be better than $0.001 \%$ at $100 \mathrm{keV}$, an impractically low figure at such voltages. Thus the electrical configuration shown in Fig. 2 was adopted for the apparatus.

The use of a common main power supply has one serious drawback: collisions in which the initial and final charge states ( $q$ and $q^{\prime}$, respectively) are unequal cannot be studied. A desire to study collisions of the type

$$
\mathrm{He}^{++}+\mathrm{H}(1 s) \rightarrow \mathrm{He}^{+}(n l)+\mathrm{H}^{+}
$$

(where $n$ and $l$ are the quantum numbers of the $\mathrm{He}^{+}$) 


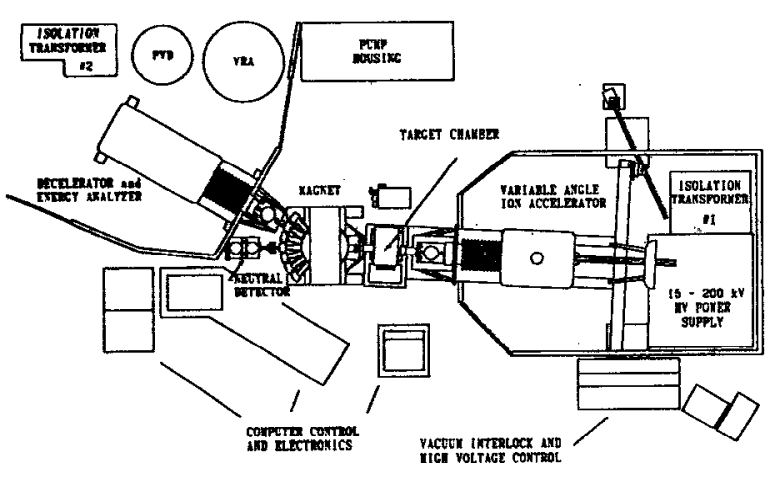

FIG. 1. Scale layout diagram of the ion-energy spectrometer at the University of Missouri-Rolla. Scattering data differential in angle are obtained by rotation of the entire ion source and accelerator about an axis through the target chamber perpendicular to the plane of the diagram. Both the acoelerator and decelerator terminals are isolated in cages during operation. The locations of the variable resistor assembly (VRA), precision voltage divider (PVD), and second isolation transformer discussed in the text are indicated.

prompted us to consider ways to modify our apparatus to allow energy spectra to be acquired for charge-changing collisions. Energy spectra for low-energy charge-changing collisions can be obtained by using separate power supplies to accelerate and decelerate the projectile ions by different voltages (see, e.g., Refs. 2 and 3). Unfortunately, this method will not work for the voltage range in which we are interested because of the power supply stability problems just mentioned. Thus an alternate solution was developed.

An electrical schematic of our modified apparatus is shown in Fig. 4. Essentially, we have inserted a highvoltage variable resistor between the decelerator and accelerator terminals, which can be used to lower the ion-source potential to a given fraction of that of the ion-energy analyzer. Because the accelerator and decelerator terminals will now be generally at significantly different potentials, two separate resistive-divider networks are needed to measure their respective voltages, and a second isolation trans-

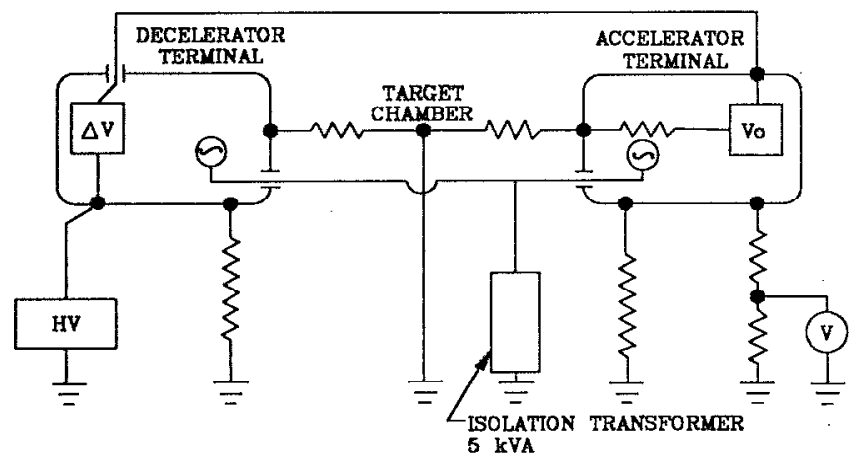

FIG. 2. Electrical schematic for the initial configuration of the ion-energy spectrometer. The main high-voltage $(\mathrm{HV})$, energy-loss $(\Delta V)$, and extraction voltage $\left(V_{0}\right)$ power supplies are indicated, as is the resistancedivider voltmeter ( $P$ ) used to measure the ion-source potential. Resistances between the target chamber and the accelerator and decelerator terminals are those of the accelerator and decelerator columns; all other sources of resistance to ground are indicated by the two remaining vertical resistance symbols.

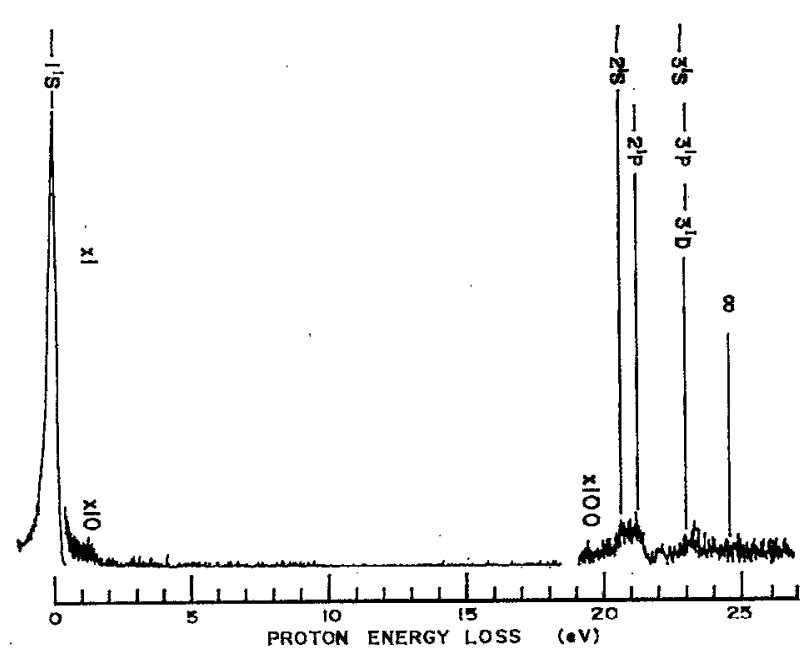

FIG. 3. Proton energy-loss spectrum for $50 \mathrm{keV} \mathrm{H}{ }^{+}+\mathrm{He}\left({ }^{1}{ }^{1} S\right)$ collisions (see Ref. 9). Peaks corresponding to excitation of $n=2$ and $n=3 \mathrm{He}$ target states, as well as that for elastic scattering, are indicater. The inelastic detector signal is multiplied by 10 or 100 .

former is required to provide power to the various components at high voltage.

An advantage of this approach is that the insensitivity of the apparatus to power supply fluctuations is preserved. Consider, as an example, an experiment involving collision (2). For the energy gain of the $\mathrm{He}^{++}$in the accelerator section to be comparable to the $\mathrm{He}^{+}$. energy loss in the decelerator, the accelerator terminal voltage must be held at half that of the decelerator terminal. This is accomplished by adjusting the variable resistor to a value equal to that between the accelerator terminal and ground. Since $q=2 e$ and $q^{\prime}=e$, the $\mathrm{He}^{+}$energy immediately after the target is equal to $2 e\left[V_{0}+1 / 2(H V+\delta+\Delta V)\right]-\Delta E$, where $\delta$ is a general, time-dependent variation in the HV supply output. The energy lost under deceleration is $e(\mathrm{HV}+\delta)$. Thus the $\mathrm{He}^{+}$energy immediately preceding the electrostatic energy analyzer is $2 e V_{0}+e \Delta V-\Delta E$ (which must equal the analyzer pass energy $E_{0}$ for the ions to be de-

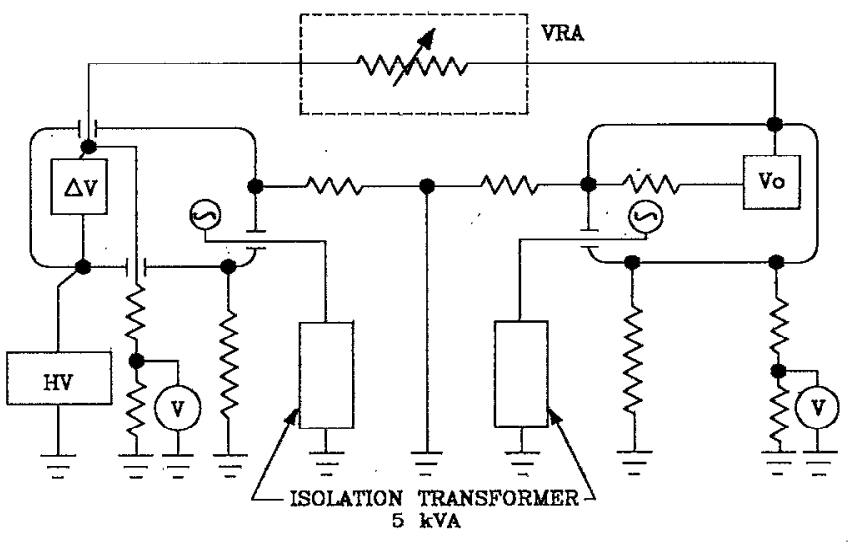

FIG. 4. Electrical schematic for the modified ion-energy spectrometer, showing the variable resistor assembly (VRA) and second isolation transformer. Two resistive-divider voltmeters $(V)$ are now used, although they are physically housed in one oil tank. 
tected), independent of any main power supply voltage fluctuations.

Such a solution is not without disadvantages. Apart from the electrical and mechanical design and construction of a high-voltage, high-precision variable resistor, the potential difficulties associated with this scheme are apparent, especially when one considers that several new possible sources of voltage fluctuation have been added to the apparatus. For example, the variable resistor and the resistors between the accelerator terminal and ground will have different temperature coefficients of resistance. Thus thermal gradients in the laboratory can, in principle, cause uncompensated beam-energy fuctuations. Projectile beamcurrent fluctuations will now result in changes in the accelerator potential, as will any corona leakage in the variable resistor or voltage divider networks. Successful implementation of a variable resistor circuit requires the resolution of these and other problems. In this report, we describe the apparatus we have used successfully to modify our ion-energy spectrometer so that charge-changing ionatom collisions can be studied.

\section{APPARATUS}

\section{A. Design considerations}

We wished to build a variable resistor that would allow us to investigate collisions in which $q / q^{\prime}$ was as high as three, such as $\mathrm{Li}^{3+}+\mathrm{X} \rightarrow \mathrm{Li}^{+}+\mathrm{X}^{2+}$. This requires a maximum resistance equal to twice $R_{A}$, the resistance between the accelerator terminal and ground. In our apparatus $R_{A}$ $=800 \pm 10 \mathrm{M} \Omega$, so we designed the variable resistor assembly (VRA) to have a maximum $2 \mathrm{G} \Omega$ impedence.

Voltage (and hence beam energy) stability was a crucial design criterion. Three potential sources of voltage fluctuations are introduced when a variable resistor and its attendant components are added to the electrical circuit. First, intermittent corona leakage from any of the new components could cause the accelerator potential, $V_{A}$, to vary. The sensitivity of $V_{A}$ to cornoa loss in the VRA, for example, depends on $R_{A}$, the VRA resistance $R_{\mathrm{VRA}}$, and the point (or points) in the VRA where the loss occurs. In a worst case $R_{\mathrm{VRA}} / R_{A}$ would equal two. Assuming that the HV power supply is not current limited, and that losses occur at the low-voltage end of the VRA, a 2 nA corona current would cause $V_{A}$ to change by a volt. In this case, 2 $\mathrm{nA}$ is less than $0.01 \%$ of the bias current through the resistors when $\mathrm{HV}=200 \mathrm{kV}$.

Consequently, every effort was made to minimize or eliminate corona currents emitted from the electrical circuitry. To this end, the geometric configuration of the apparatus was dictated primarily by considerations relating to the minimization of electric field throughout the design. Our HV power supply and accelerator and decelerator terminal configurations limit us to a maximum high voltage of $200 \mathrm{kV}$. We assumed that electrical components at high voltage would be submersed in dielectric oil (Shell DIALA-AX) with a minimum dielectric breakdown strength of about $140 \mathrm{kV} / \mathrm{cm}$. As a design goal, we took 30 $\mathrm{kV} / \mathrm{cm}$ as a maximum allowable electric field. While this value was somewhat arbitrary, its choice was guided in part by a tendency for field-aided emission from electrodes to begin at about one-fifth of the breakdown field, ${ }^{15}$ and in part by our observation that this seemed to be a much more conservative value than that employed in the design of many pieces of oil-immersed high-voltage apparatus with which we were familiar.

A second, related source of fluctuation in $V_{A}$ is variation in ion-beam current from the source. When $V_{A}$ is maintained directly by high-voltage power supplies, as in the configuration shown in Fig. 2, this is not a problem. In a divider configuration, however, changes of $1 \mathrm{nA}$ in beam current can cause $V_{A}$ to vary by large fractions of a volt. Unfortunately, such effects are unavoidable, and care must simply be taken to keep beam-current fuctuations as small as possible.

Finally, variation in $V_{A}$ can result from timedependent temperatures and/or temperature gradients in the lab. Since

$$
V_{A}=(\mathrm{HV})\left[\frac{R_{A}}{\left(R_{A}+R_{\mathrm{VRA}}\right)}\right]
$$

any change in the relative values of $R_{A}$ and $R_{\text {VRA }}$ due to temperature-dependent resistance variations, after $R_{\text {VRA }}$ has been initially set, will cause drifts in $V_{A}$. To minimize these, the composite temperature coefficients of resistance of $R_{A}$ and $R_{\text {VRA }}$ should be as close as possible. Alternatively, the VRA could be temperature-stabilized relative to the accelerator resistors, or adjusted on a continuous basis to compensate for temperature variations.

A last significant design concern was the possible effect of voltage coeficients of resistance on comparative measurements of accelerator and decelerator terminal potentials. We decided to construct two matched precision voltage divider (PVD) strings to measure $V_{A}$ and the decelerator potential, $H V$. The voltage readout from these were used to set $R_{\mathrm{VRA}}$ for a given experiment. As we shall discuss in Sec. III A, a standard cross section measurement first involves the transmission through the entire spectrometer of a beam of "final state" ions $\left[\mathrm{He}^{+}\right.$in the case of reaction (2)] with the accelerator and decelerator terminals at the same potential $\left(R_{\mathrm{VRA}}=0\right)$. Following this, $R_{\mathrm{VRA}}$ is increased until $R_{A} /\left(R_{\mathrm{VRA}}+R_{A}\right) \approx q^{\prime} / q$, as determined from the PVD readouts. When the voltage across the accelerator divider string decreases, the resistance ratio of the main voltage dropping resistor $(R)$ to the resistor across which the readoul voltmeter is placed $\left(R_{V}\right)$ will change if the voltage coefficients of resistance differ for the two resistances or, if they are equal, when the individual component resistors making up $R$ have different voltage drops across them (i.e., have different resistances) than does $R_{V}$.

This problem can be illustrated by considering worstcase experiments where $q / q^{\prime}=3$. It is desirable for $(R$ $+R_{V}$ ) to be comparable to or larger than $R_{A}$ to minimize current (and the attendant ripple, proportional to current) drawn from the HV power supply as well as power dissi. pation in the PVD strings. Conversely, $R_{V}$, and hence $R$, should not be so high that the input impedance of the 
voltmeter affects the PVD readout. (This latter problem cannot be solved by the use of small values of $R_{V}$ without running the risk of reducing the readout voltage to unacceptably low levels.) Consider $R=10 \mathrm{G} \Omega$, comprising ten $1 \mathrm{G} \Omega$ resistors in series, and $R_{V}=1 \mathrm{M} \Omega$ using a single resistor. If we assume that individual resistors have the same voltage coefficients of resistance, $C_{V}$, equal to -1 $\mathrm{ppm} / \mathrm{V}$, and that $R$ and $R_{V}$ are the resistance values with $200 \mathrm{kV}$ across the PVD string, corresponding to approximately 20000 and $20 \mathrm{~V}$ across the $1 \mathrm{G} \Omega$ and $1 \mathrm{M} \Omega$ resistors, respectively, then when $V_{A}=66.7 \mathrm{kV}$ the ratio $R_{V} / R$ $=0.98 \times 10^{-4}$, as opposed to $1.00 \times 10^{-4}$. This corresponds to a PVD reading error for $V_{A}$ of $\approx 1300 \mathrm{~V}$. Since it is impractical to search for a (possibly very small) chargetransfer peak in an ion-energy spectrum by varying $\Delta V$ over a range greater than a few hundred volts, a reading error this large, which would result in a correspondingly inaccurate setting of $V_{A}$, is unacceptable. Moreover, $C_{V}$ generally increases monotonically with resistance value, which exacerbates this problem.

The problem of voltage coefficients of resistance are minimized first, obviously, by selecting resistors with low values of $C_{V}$. Additionally, it is advantageous to have as many individual resistors as possible make up the composite resistance $R$, because this minimizes the voltage drop across, and power dissipation in, a given resistor. The number of resistors cannot be so large, however, that fields between the corona caps placed at the resistor junctions exceed the design limit. Our design goal was to achieve PVD accuracies at all voltages of one part in $10^{3}$, which is somewhat better than the specified accuracies of commercial devices, ${ }^{16}$ but significantly worse than those claimed by some authors in the literature. ${ }^{17,18}$ An accuracy of $0.1 \%$ would guarantee that $\Delta V$ would not have to be varied by more than $100 \mathrm{~V}$ to detect $q^{\prime}$ ions once $R_{\mathrm{VRA}}$ had been set. (In principle, $R_{\mathrm{VRA}}$ could be varied to search for chargetransfer peaks, but this would be more difficult; see Secs. II B and III A.)

Finally, it is important to remember that the considerations discussed earlier regarding corona currents and temperature coefficients of resistance apply, at least partially, to the design of the PVD as well as that of the VRA.

\section{B. Electrical and mechanical design}

In this section, we discuss in detail the electrical and mechanical construction of the variable resistor assembly (VRA) and precision voltage divider (PVD), the ancillary electrical equipment needed for the modification, and several aspects of the installation of this apparatus.

\section{Variable resistor assembly (VRA)}

The VRA is adjustable from a shorted position, with zero resistance, to a maximum value of about $2050 \mathrm{M} \Omega$. It comprises three subassemblies, each with variable resistance: (1) A "coarse-adjustment stack", consisting of one hundred $20 \mathrm{M} \Omega$ resistors in series, which are in turn divided into two sets of 50 resistors each. These individual sets are mounted in a zig-zag fashion around the periphery of each of two plexiglass cylinders $81.3 \mathrm{~cm}$ in diameter

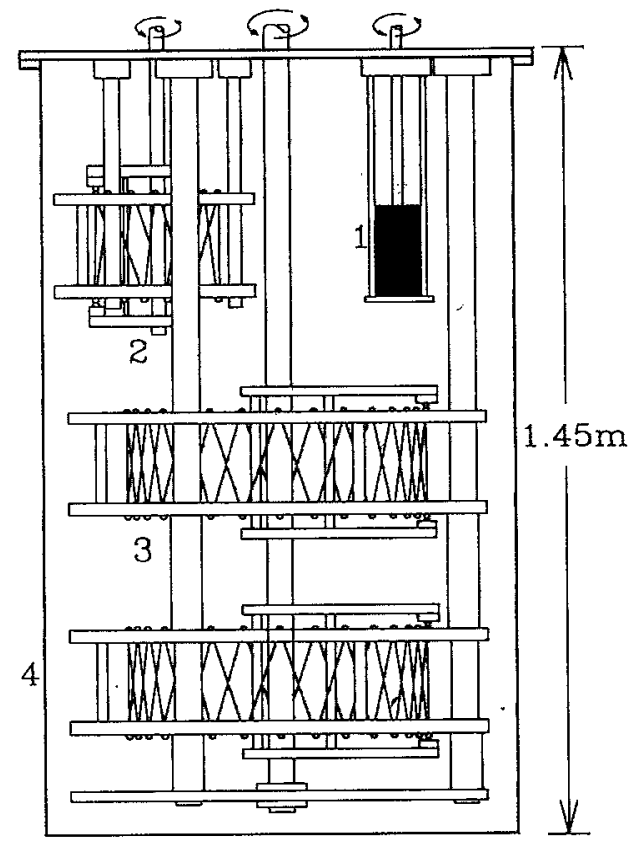

FIG. 5. Scale assembly drawing of the variable resistor assembly (VRA). Shown are (1) fine adjustment (40-turn wire-wound $2.5 \mathrm{M} \Omega$ potentiometer); (2) intermediate-adjustment resistor stack ( $24-2 \mathrm{M} \Omega$ resistors and rotary wiper); (3) two coarse-adjustment resistor stacks (each consisting of 50-20 M $\Omega$ resistors and rotary wiper); (4) oil tank. The rotary wipers are turned by plexiglass rods as indicated. For simplicity, cable connections between the stacks and potentiometer, and external cable connections and clamps are not shown. The surface of the oil in the tank is about $2 \mathrm{~cm}$ below the lid.

(Fig. 5). (2) An "intermediate adjustment stack" consisting of twenty-four $2 \mathrm{M} \Omega$ resistors, mounted similarly to those of the coarse stack around a $39.4 \mathrm{~cm}$ diam plexiglass cylinder. (3) A "fine adjustment" resistor, which is a 40turn $2.5 \mathrm{M} \Omega$ potentiometer. All three subassemblies are housed in a plexiglass support structure, which is suspended from the lid of a mild steel oil tank $104 \mathrm{~cm}$ in diameter and $145 \mathrm{~cm}$ high. The tank, manufactured by the Nooter Corporation, holds about 300 gal of Shell DIALA-AX dielectric insulating oil with the VRA components in place.

The three resistor subassemblies are connected in series between the accelerator and decelerator terminals, as shown in Figs. 4 and 6. The series resistance of the coarse and intermediate stacks is varied by sliding spring-loaded polished steel makc-beforc-break wipers over corona caps at the junctions of the individual resistors in the stacks (Fig. 7). These corona caps are arranged in a circular pattern. The wipers are mounted in radial plexiglass arms connected to vertical shafts which pass through the centers of the resistor stacks. The wipers can thus be moved by rotating the shafts, which emerge through the oil tank lid. A similar shaft is attached directly to the fine-adjustment resistor.

The geometry, dimensions, placement, and wiring of the resistor stacks were carefully analyzed to minimize electric fields. The resulting quasicylindrical geometry was considered the best design both for mechanical wiper motion and field reduction. The highest fields in the tank oc- 


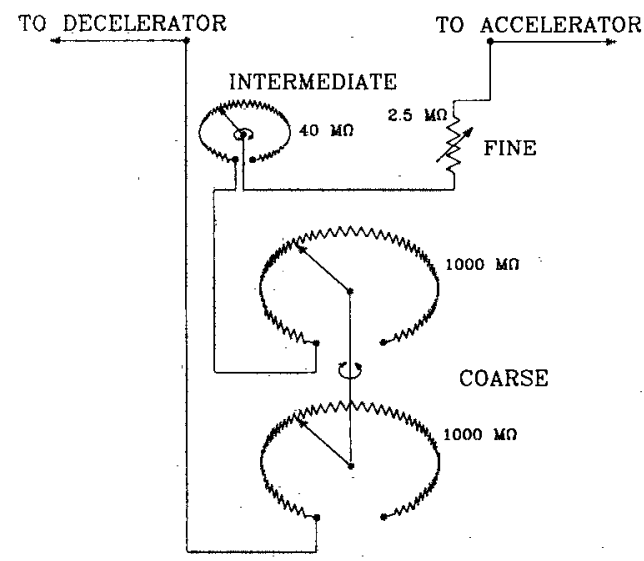

FIG. 6. Electrical schematic of the variable resistor assembly (VRA).

cur near the corona cap terminating the high-voltage ends of the two coarse-adjustment stacks. Approximating the caps as spheres $1.9 \mathrm{~cm}$ in diameter, the field at the surface of an isolated cap at $200 \mathrm{kV}$ is $210 \mathrm{kV} / \mathrm{cm}$, or about a factor of 7 too large for our design limit. By placing caps in the stack along a circle segment, the maximum field is reduced dramatically. If the angle subtended by the segment becomes too large, however, fields along the chord between the corona caps at the high-and low-voltage ends of the string increase prohibitively. Another large reduction in the fields near the high-voltage corona cap is achieved by placing "dummy" corona caps on the circle between the stack's high- and low-voltage ends. These are maintained at incremental, intermediate voltages by connecting them to corresponding resistor junctions across the

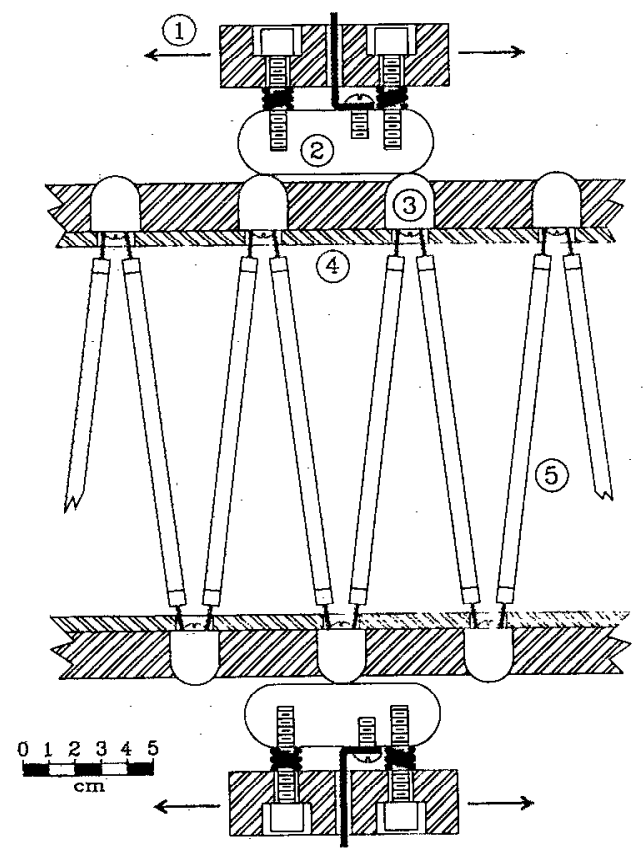

FIG. 7. Detail of coarse-adjustment resistor stack periphery showing make-before-break spring-loaded wipers: (1) cross-sectional view of rotating plexiglass wiper arm; (2) spring-loaded wiper with electrical connection; (3) corona caps; (4) two-piece plexiglass corona cap disks; (5) metal-oxide-film resistors:

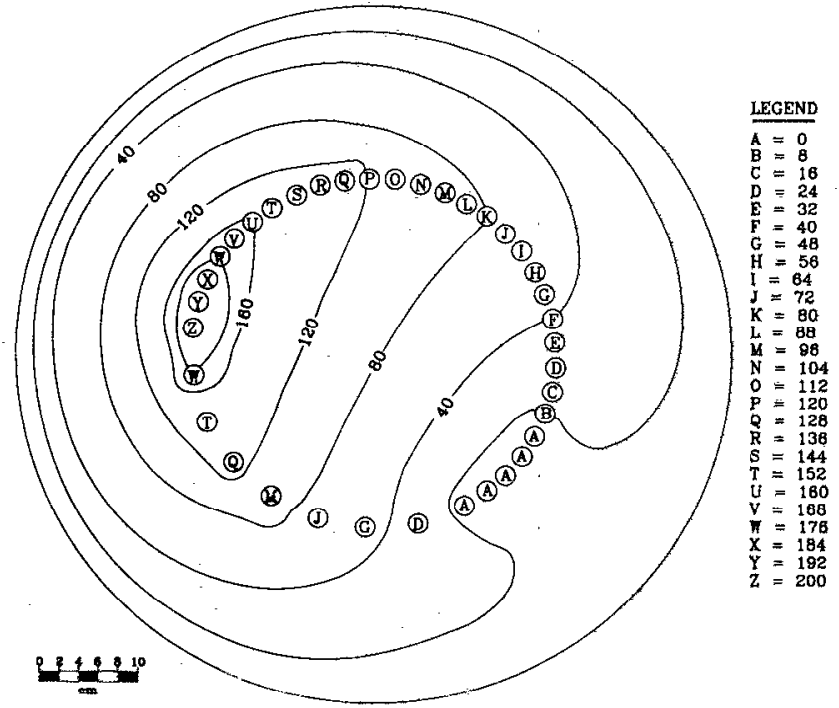

FIG. 8. Precision voltage divider (PVD) potential surfaces calculated for the case of no oil in the tank $(\epsilon=1)$ by solving Laplace's equation in two dimensions using the finite-element relaxation technique. The dielectric effects of the plexiglass support structurc have been neglected. Because oil and plexiglass have similar dielectric constants, these potential curves should be fairly representative of the equivalent curves when the tank is filled with oil. An input potential of $200 \mathrm{kV}$ is assumed. Coronia caps, 1.9 $\mathrm{cm}$ in diameter, have centers placed at $8^{\circ}$ or $16^{\circ}$ intervals on a $37.5 \mathrm{~cm}$ diam circle. These caps are labeled with their respective voltages (in units of $\mathrm{kV}$ ); the caps labeled to be at zero, the value used in the calculation, are actually at potentials of less than $50 \mathrm{~V}$ associated with the $2 \mathrm{M} \Omega$ voltage-sensing resistors (see Fig. 9 and the text). Corona caps with $16^{\circ}$ gaps between them are "dummy" electrodes used to reduce internal electric fields in the design. The outer circular boundary is the oil tank, taken to be at ground.

diameter of the circle. This basic arrangement is illustrated in Fig. 8. (Figure 8 actually shows a resistive stack in the PVD; see Sec. III $\mathrm{B} 2$ below. The equipotential contours in Fig. 8 were calculated by solving Laplace's equation in two dimensions for the potential distributions indicated.)

The electrical configuration shown in Fig. 6 also serves to minimize electric fields because equipotential points on the two strings are vertically above each other in the tank. Moreover, resistors and caps not in series in the circuit "float up" to approximately $V_{A}$, which further decreases voltage differences across the stack's horizontal dimensions. Finally, the intermediate and fine-control resistors are placed near the low-voltage end of the coarse stack.

Given the VRA configuration shown in Figs. 5 and 6, our best estimate of the maximum electric field in the VRA with $H V=200 \mathrm{kV}$ and no oil in the tank is $\approx 24 \mathrm{kV} / \mathrm{cm}$. This field, which is a factor of 5.9 below the breakdown field for DIALA-AX oil, is at the surface of the $200 \mathrm{kV}$ corona cap at the bottom of the coarse stack and points toward the bottom corner of the oil tank.

The dielectric strength of insulating oil can be severely compromised by particulate and chemical contaminants. ${ }^{15,19}$ We took a number of precautions to reduce such contamination to a minimum. The oil tank and all internal components were cleaned with soap and water (for plastic) or organic solvents and then rinsed with methanol. Latex gloves were worn during all assembly procedures. 
Dust is prevented from easily entering the tank with a combination of foam-rubber seals and tightly constricted or covered openings.

The inevitable arcing that occurs between the sliding contacts and the resistor chain when $R_{\mathrm{VRA}}$ is changed acts to break down the oil into components with lower dielectric strength, and will cause bubble formation which also reduces the minimum breakdown field. To reduce arcing as much as possible, the sliding wiper was made long enough that it can touch two adjacent corona caps simultaneously ("make-before-break"), and the number of resistors in the coarse stack was chosen to be as large as possible, consistent with the requirement of minimizing electric fields between the caps.

The discrete resistors used for the coarse and intermediate stacks are of the metal-oxide film type specifically designed for oil bath operation (Dale ROX-6). Units with maximum power and voltage ratings $(10 \mathrm{~W}$ and $45 \mathrm{kV}$, respectively) were chosen to minimize their voltage coefficients and temperature increase under load. ${ }^{20}$ (The maximum steady-state power dissipation in the $20 \mathrm{M} \Omega$ resistors is $0.18 \mathrm{~W}$.) The temperature coefficients of resistance for all resistors are less than $50 \mathrm{ppm} /{ }^{\circ} \mathrm{C}$ and are typically about $20 \mathrm{ppm} /{ }^{\circ} \mathrm{C}$ (these values were measured for individual resistors by the manufacturer). Because these values did not match those of the carbon-film resistors already in place on the accelerator, which had estimated values of $\approx 500 \mathrm{ppm} /{ }^{\circ} \mathrm{C}$, we considered the options of temperature stabilization of the VRA oil, and active control of the fine and intermediate resistors to compensate for temperature drifts. The former possibility was not pursued because of concerns about convective stirring of particulate contaminants which would otherwise settle to the bottom of the oil tank, and which could reduce the oil's dielectric strength. The second option was developed but did not need to be implemented (see Secs. II B 2 and III A).

Fine resistance adjustment is provided by a Beckman Helipot Series E $2.5 \mathrm{M} \Omega$ 40-turn wire-wound potentiometer rated at $20 \mathrm{~W}$ and $1000 \mathrm{~V}$. Although this unit was not certified by Beckman to be oil compatible, we found it to be so. Two $6 \mathrm{~mm}$ holes were drilled in the top and bottom of the phenolic case to permit free circulation of oil over the windings. The potentiometer's coefficient of resistance is comparable to those of the fixed resistors $\left(\approx 20 \mathrm{ppm} /{ }^{\circ} \mathrm{C}\right)$.

All structural components in the oil tank are made of plexiglass, with the exception of a few PVC nuts. (Nylon components were not used because of their tendency to absorb water.) Plexiglass has a somewhat higher dielectric strength $(\approx 160 \mathrm{kV} / \mathrm{cm})$ than does DIALA-AX. The two coarse stacks as a single unit, the intermediate stack, and the find adjustment potentiometer are each supported independently from the lid by solid plexiglass rods (Fig. 5). The resistance of each subsection is adjusted by rotation of rods which emerge from the lids and are coupled to stepping motors. In the case of the coarse and intermediate stacks, right-angle gear reducers are used to increase the torque available to turn the wiper arms.

The high-voltage cables leading into and out of the VRA are rated at $300 \mathrm{kVDC}$ and have a semiconductor

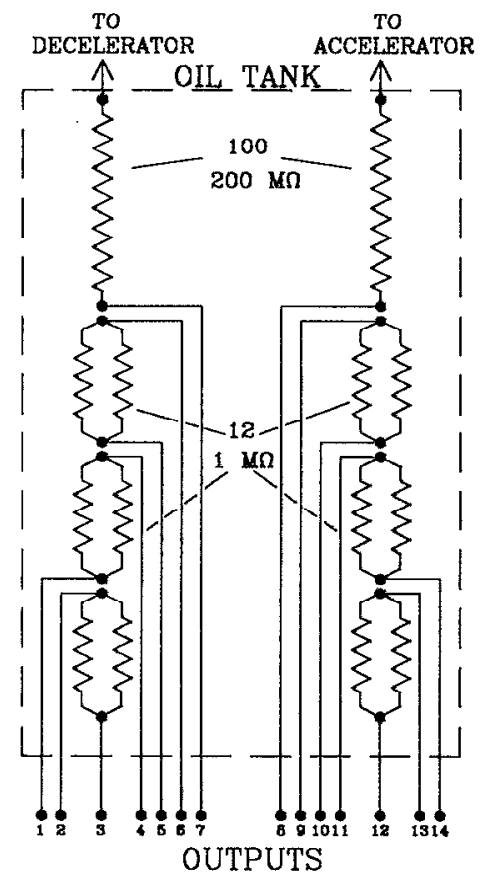

FIG. 9. Electrical schematic of the precision voltage divider (PVD; see text).

core shield for corona suppression (Dielectric Sciences \#2077). They have a $3.8 \mathrm{~cm}$ outside diameter and are mechanically restrained by aluminum clamps mounted on the lid.

\section{Precision voltage divider (PVD)}

The PVD and VRA are similar in many respects. Both accelerator and decelerator PVD strings are housed in an oil tank $102 \mathrm{~cm}$ high and $79 \mathrm{~cm}$ in diameter. A common oil bath is used to minimize temperature-related voltage measurement errors. Each string has 50-200 M $\Omega$ and 6-2 M $\Omega$ metal-oxide film resistors (Dale ROX-6). The $200 \mathrm{M} \Omega$ resistors are in series and the $2 \mathrm{M} \Omega$ resistors are connected in parallel to form three $1 \mathrm{M} \Omega$ composite resistors in each string (Fig. 9). The two strings are mounted around the periphery of $40.6 \mathrm{~cm}$ diam plexiglass "stacks" in the same fashion as the discrete resistors in the VRA. The stacks are supported from the tank lid by three plexiglass rods. In order to reduce electric fields, corona caps at intermediate voltages are placed on the circle segment between the highand low-voltage ends of the strings (Fig. 8). We estimate that the maximum field in the PVD tank with no oil at 200 $\mathrm{kV}$ is $30 \mathrm{kV} / \mathrm{cm}$. This value is somewhat higher than the corresponding maximum in the VRA because the PVD tank is smaller than that of the VRA, and the voltage drop across a given stack is a full $200 \mathrm{kV}$ in the PVD, instead of $133 \mathrm{kV}$.

The $1 \mathrm{M} \Omega$ composite resistors at the low-voltage ends of the strings are connected in a way that allows PVD readings to be taken in one of two modes (Fig. 9). In the standard readout mode, two Fluke 8840A 5-1/2 digit voltmeters are connected across each of the bottom $1 \mathrm{M} \Omega$ resistors (connections 1, 2, 13, and 14). A multiturn precision potentiometer can be inserted in either the acceler- 
ator or decelerator strings. When the VRA is shorted, the potentiometer is adjusted to make the two Fluke readings the same. The two output voltages are then used to set the VRA with $1 \mathrm{~V}$ ( 1 digit) precision. The PVD resistances were chosen such that the $20 \mathrm{~V}$ scale could be used on the Flukes, giving these devices an input impedence $>10^{10} \Omega$ with correspondingly negligible loading error on the PVD readout. A second possible readout method, which we have not used to date, would involve measurement of current through a shunt connecting the two divider strings. In a $q / q^{\prime}=3$ experiment, for example, an ammeter would be placed between connections $1 / 2$ and $8 / 9$ (Fig. 9), and the VRA would be set in correspondence with a null reading of the meter. As can be seen from Fig. 4, this null reading would be unaffected by changes in $\Delta V$. The output from the ammeter could also be used as feedback to a steppermotor driver which controlled the VRA. In such a manner, temperature drifting between $R_{A}$ and $R_{\mathrm{VRA}}$ could be compensated. This latter readout technique has the advantage that errors due to finite input impedence of a readout voltmeter are eliminated.

The high-voltage connections from the accelerator and decelerator are made to the PVD stacks in the same manner as the VRA, using $300 \mathrm{kV}$ cable. External connections to the low-voltage node points (1-14 in Fig. 9) are made using $40 \mathrm{kV}$ cable (Belden 8866) connected to SHV bulkhead connectors mounted on the PVD lid. Care was taken to keep these latter cables in regions of the tank at relatively low potential.

\section{Installation}

Modification of the ion-energy spectrometer occurred in three steps. First, the isolation transformer (Del 3CTR250-21; $3 \mathrm{kVA}$ at $250 \mathrm{kV}$ ) was installed. As shipped, it had a two-leg, two-cable high-voltage power line emerging from its oil tank. We replaced this line with a single, coaxial $250 \mathrm{kV}$ power line (Chester Cable Corp. \#1965, which is no longer manufactured) to avoid any $60 \mathrm{~Hz}$ magnetic fields which would result from a biaxial arrangement. The VRA and PVD were then attached to the system. The new connections required approximately $200 \mathrm{ft}$ of high-voltage cable. Care was taken to run the cable along existing resistor-graded potential paths (accelerator/ decelerator columns or supports) when its outer-casing potential went from ground to high voltage. Finally, voltage breakdown tests and the last mechanical tests were made prior to putting oil in the tanks. No arcing problems were noted up to terminal voltages of $75 \mathrm{kV}$. The PVD and VRA tanks were then filled using a clean polyethylene hand pump.

\section{EXPERIMENTAL PROTOCOL AND RESULTS}

In our initial experiments with the VRA, we considered only the reaction

$$
\mathrm{He}^{++}+\mathrm{He}\left(1^{1} S\right) \rightarrow \mathrm{He}^{+}(n)+\mathrm{He}\left(n^{\prime}\right) .
$$

Since $q / q^{\prime}=2$ in this collision, we modified the connections of the VRA coarse stacks to further reduce electric field

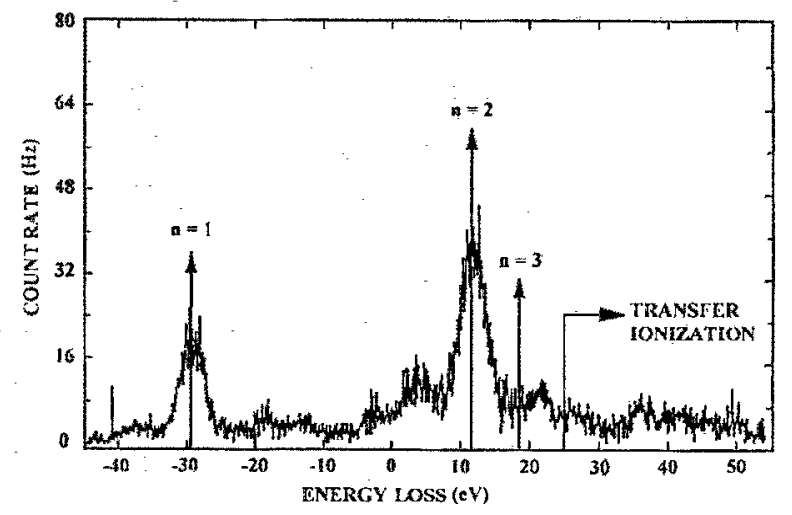

FIG. 10. Energy gain/loss spectrum for single-electron capture by $90 \mathrm{keV}$ $\mathrm{He}^{+}+$incident on a ground-state helium target (collision 4 ). The energygain peak at the left $(-29 \mathrm{eV}$ energy loss) corresponds to superelastic scattering into the projectile $\mathrm{He}^{+}(n=1)$ state, while the major central energy-loss peak is associated with either the projectile or target ion in an $n=2$ state, with the partner ion in its ground state. The $n=3$ energy-loss position and transfer ionization limits are indicated as well (see text).

stress in the oil tank during switching. This was accomplished by bypassing the upper coarse stack altogether, meaning that coarse resistor increments were now 20 instead of $40 \mathrm{M} \Omega$ (Fig. 6). The upper stack was connected electrically to the low-voitage end of the lower stack.

An ion-energy spectrum for collision (4) is taken as follows. (For this discussion, we will assume $100 \mathrm{keV}$ $\mathrm{He}^{++}$projectiles.) The apparatus downstream from the target is first tuned for transmission of a $\mathrm{He}^{+}$beam. With the VRA shorted and $\mathrm{HV}=100 \mathrm{kV}, \mathrm{a} \mathrm{He}^{+}$beam from the ion source is selected by a Wien filter in the accelerator terminal. This beam is accelerated and directed through the (empty) target cell, and is then guided into the decelerator where it is focused and steered to the ion detector following energy analysis. Once the detected $\mathrm{He}^{+}$signal has been optimized, the Wien filter fields are changed to select $\mathrm{He}^{++}$, and the VRA is unshorted and adjusted so that the accelerator voltage, as read by the appropriate PVD voltmeter, is half $(50 \mathrm{kV})$ that of the decelerator. At this point, the $\mathrm{He}^{++}$beam is directed onto the He target, and the $\Delta V$ power supply (or, alternately, the fine control of the VRA) is adjusted to search for a $\mathrm{He}^{+}$signal corresponding to single-electron capture from the target by $\mathrm{He}^{++}$ions.

A spectrum thus obtained, with $90 \mathrm{keV} \mathrm{He}{ }^{++}$, is shown in Fig. 10. The peak at the left corresponds to superelastic capture into the $\mathrm{He}^{+}(n=1)$ state. Most capture, associated with the large central peak, corresponds to an $n=2$ state of either the projectile or ionized target and an $n=1$ state of the other collision partner. Neither collisions involving higher levels of either the projectile or target ions, nor transfer-ionization processes, in which the target is doubly ionized with one of the electrons placed in a continuum state, are readily observable. The two features at about 3 and $20 \mathrm{eV}$ of energy loss do not correspond to any know collision process, and are thought to result from spurious reflections in the ion-energy analyzer.

In practice, we have found that the procedure dis- 
cussed above, in particular steps involving the VRA and PVD, are (somewhat surprisingly) straightforward. Once the VRA is set, we observe no variations of the PVD voltmeter readouts by more than half a digit, or about $1 \mathrm{~V}$, over periods approaching $1 \mathrm{~h}$. Thus temperature-related resistance fluctuations in $R_{A}$ and $R_{\mathrm{VRA}}$ (or the PVD divider strings) appear to be quite small. This obviates the need for temperature control of the lab or the resistors themselves, or active feedback adjustment of the VRA. Short-term variations, which might be caused, for example, by unstable corona discharge, have not been observed either.

We find that the system stability and the controls of the VRA are such that we can obtain any desired reading of the accelerator PVD voltmeter at will, precise to the last digit, or $\pm 1 \mathrm{~V}$. We also find, however, that upon setting the VRA resistance to the value where, e.g., the main $n=2$ charge-transfer peak should be observed, that the $\Delta V$ power supply must typically be adjusted to negative values by between 20 and $50 \mathrm{~V}$ before $\mathrm{He}^{+}$ions are detected. This is presumably due in large part to the voltage coefficient effect discussed earlier. The $200 \mathrm{M} \Omega$ PVD resistors have voltage coefficients of resistance of about $-1.3 \mathrm{ppm} / \mathrm{V} .^{21}$ Thus, when $V_{A}$ is dropped from, e.g., 100 to $50 \mathrm{kV}$, the accelerator PVD string resistance increases by roughly one part in $10^{3}$, or about $10 \mathrm{M} \Omega$. Thus the accelerator voltmeter will read approximately $50 \mathrm{~V}$ low. Other reading errors could be caused by steady-state current leakage between the resistor strings in the PVD through the oil, or from the strings to ground, or by temperature variations between the PVD strings. We calculate both of these effects to be much smaller, however, than that due to the voltage coefficients of resistance.

\section{ACKNOWLEDGMENTS}

We would like to thank D. M. Blankenship, J. D. Morgan, E. R. Redd, and especially J. T. Park for useful discussions. C. A. McWhorter built much of the VRA and PVD, and B. W. Stiritz performed the Laplace equation calculations. This work was supported by the U.S. Department of Energy Office of Fusion Energy under Grant No. DE-FG02-84ER53188.

I J. T. Park, Adv. At. Mol. Phys. 19, 67 (1983).

${ }^{2}$ J. H. Moore, Jr., J. Chem. Phys. 55, 2760 (1971).

${ }^{3}$ M. Lennon, R. W. McCullough, and H. B. Gilbody, J. Phys. B 16, 2191 (1983).

${ }^{4}$ V. V. Afrosimov, A. A. Basalaev, G. A. Leiko, and M. N. Panov, Sov. Phys. JETP 47, 837 (1978).

${ }^{5}$ E. H. Nielsen, L. H. Anderson, A. Bárány, H. Cederquist, J. Heinemeier, P. Hvelplund, H. Knudsen, K. B. MacAdam, and J. Sorensen, J. Phys. B 18, 1789 (1985).

${ }^{6}$ S. Ohtani, Y. Kaneko, M. Kimura, N. Kobayashi, T. Iwai, A. Matsumoto, K. Okuno, S. Takagi, H. Tawara, and S. Tsurubuchi, J. Phys. B 15, L533 (1982).

${ }^{7}$ For further references to projectile, or translational, ion-energy spectroscopy, see, e.g., R. K. Janev and H. Winter, Phys. Rep. 117, 265 (1985), and C. L. Cocke and R. E. Olson, ibid. 205, 154 (1991).

${ }^{8}$ See, e.g., Y. P. Chong and W. L. Fite, Phys. Rev. A 16, 933 (1977).

${ }^{9}$ T. J. Kvale, D. G. Seely, D. M. Blankenship, E. Redd, T. J. Gay, M. Kimura, E. Rille, J. L. Peacher, and J. T. Park, Phys. Rev. A 32, 1369 (1985).

${ }^{10}$ See, e.g., R. Hoekstra, F. J. deHeer, and R. Morgenstern, J. Phys. B 24, 4025 (1991).

${ }^{11}$ D. H. Crandall, J. A. Ray, and C. Cisneros, Rev. Sci. Instrum. 46, 562 (1975), and references therein.

${ }^{12}$ J. T. Park and F. D. Schowengerdt, Rev. Sci. Instrum. 40, 753 (1969).

${ }^{13}$ J. T. Park and F. D. Schowengerdt, Phys. Rev. 185, 152 (1969).

${ }^{14}$ J. T. Park, J. M. George, J. L. Peacher, and J. E. Aldag, Phys. Rev. A 18, 48 (1978).

${ }^{15}$ T. J. Lewis, in IIigh Voltage Technology, edited by L. L. Alston (Oxford, London, 1968), Chap. 6.

${ }^{16} \mathrm{High}$-voltage dc resistive-divider networks with specified accuracies of between $3 \%$ and $0.25 \%$ are available from, e.g., Glassman High Voltage, Universal Voltronics, Spellman High Voltage, Hippotronics, and Del Electronics.

${ }^{17}$ T. B. Rymer and K. H. R. Wright, J. Sci. Instrum. 29, 139 (1952).

${ }^{18}$ J. H. Park, J. Res. Natl. Bur. Stand. C 66, 19 (1962).

${ }^{19}$ See, e.g., E. Kuffel and M. Abdullah. High-Voltage Engineering (Pergamon, Oxford, 1970) and also Chap. 7 of Ref. 15 (written by Z. Krasucki).

${ }^{20}$ Dale ROX metal-oxide resistors have negative voltage coefficients of resistance which are roughly inversely proportional to their voltage ratings, and whose absolute values increase approximately as the onethird power of their resistances.

${ }^{21}$ This value is obtained by the linear extrapolation of values for average voltage coefficients of resistance quoted by the manufacturer for metaloxide-film resistors with lower voltage and power ratings (Dale catalog Vol. 4, rev. 829, p. 185). See also Ref. 20. 